\title{
Optimal topology design of structures under dynamic loads*
}

\author{
S. Min and N. Kikuchi \\ Department of Mechanical Engineering and Applied Mechanics, The University of Michigan, Ann Arbor, MI 48105, USA
}

\author{
Y.C. Park, S. Kim and S. Chang \\ LG Production Engineering Research Center, 19-1 Cheongho-Ri Jinwuy-Myon Pyungtaek-Si Kyunggi-Do 451-860, Korea
}

\begin{abstract}
When elastic structures are subjected to dynamic loads, a propagation problem is considered to predict structural transient response. To achieve better dynamic performance, it is important to establish an optimum structural design method. Previous work focused on minimizing the structural weight subject to dynamic constraints on displacement, stress, frequency, and member size. Even though these methods made it possible to obtain the optimal size and shape of a structure, it is necessary to obtain an optimal topology for a truly optimal design. In this paper, the homogenization design method is utilized to generate the optimal topology for structures and an explicit direct integration scheme is employed to solve the linear transient problems. The optimization problem is formulated to find the best configuration of structures that minimizes the dynamic compliance within a specified time interval. Examples demonstrate that the homogenization design method can be extended to the optimal topology design method of structures under impact loads.
\end{abstract}

\section{Introduction}

In the past, most efforts in the field of optimal design of structures have focused on solving optimum structural problems for static loads. Many problems, however, require a dynamic analysis to predict the displacement and stresses in the structure. Compared to static load problems dealing with the steady state response, the dynamic load problem is concerned with the transient response, which depends on an additional parameter--time. The optimal design problem of elastic structures under dynamic loads has received little attention due to complexities associated with the dynamic load. However, many techniques developed for the static load problems can be used to solve the dynamic load problems.

When elastic structures are subjected to dynamic loads, their shape and topology determine the dynamic characteristics of structures such as dynamic displacement and stress concentration. Thus, it is important to establish an optimal design method to generate structures which produces better dynamic performance. Therefore, the challenge is to apply the homogenization design method to a structural topology design problem subjected to dynamic loads.

One of the earliest works on structural optimization under dynamic loads can be found in the paper by Fox and

\footnotetext{
* Presented at WCSMO-2, held in Zakopane, Poland, 1997
}

Kapoor (1970). They formulated the minimum weight design problem of frame structures subject to base motion and constraints on dynamic stresses and displacements, and obtained the solution by using a mathematical programming approach. A literature survey of optimal structural design under dynamic constraints was presented by Pierson (1972). In this review, dynamic constraints are classified as constraints on structural frequencies and on quantities directly related to the dynamic response. The optimal design of structures subjected to dynamic loads was undertaken by Cassis and Schmit (1976) for minimum weight design of orthogonal planar frames. In this work, time-dependent dynamic response quantities were included in the problem using the approximation concepts, and the disjoint nature of the feasible region in the design space was discussed. The optimum structural design for dynamic response constraints was presented by Yamakawa (1984), in which the dynamic response peaks were considered as the objective function. To deal with large-scale mechanical and structural systems subjected to dynamic loads, Chahande and Arora (1994) applied the multiplier method to a large-scale dynamic response structural optimization problem. An augmented Lagrangian was constructed to treat all the constraints and the cost function as one equivalent functional to be minimized, which results in significant savings in computational efforts. Previous work focused on minimizing the structural weight subject to dynamic constraints on displacement, stress, frequency, and member size. Even though these methods made it possible to obtain the optimal size and shape of a structure, it is necessary to obtain an optimal topology for a truly optimal design.

In this paper, the homogenization design method is utilized to generate the optimal topology of structures subjected to impulsive-type dynamic loadings. A finite element model of the structural response is employed to solve the linear dynamic problem since analytical methods for dynamic analysis and design are impractical. An explicit direct integration scheme with the central difference method is implemented to solve the linear transient structural analysis because the interest lies in the dynamic response within a very short period. The optimization problem is formulated to find the material distribution that minimizes the mean dynamic compliance of a structure within the specified time interval subject to the total mass constraint, size and orientation design vari- 


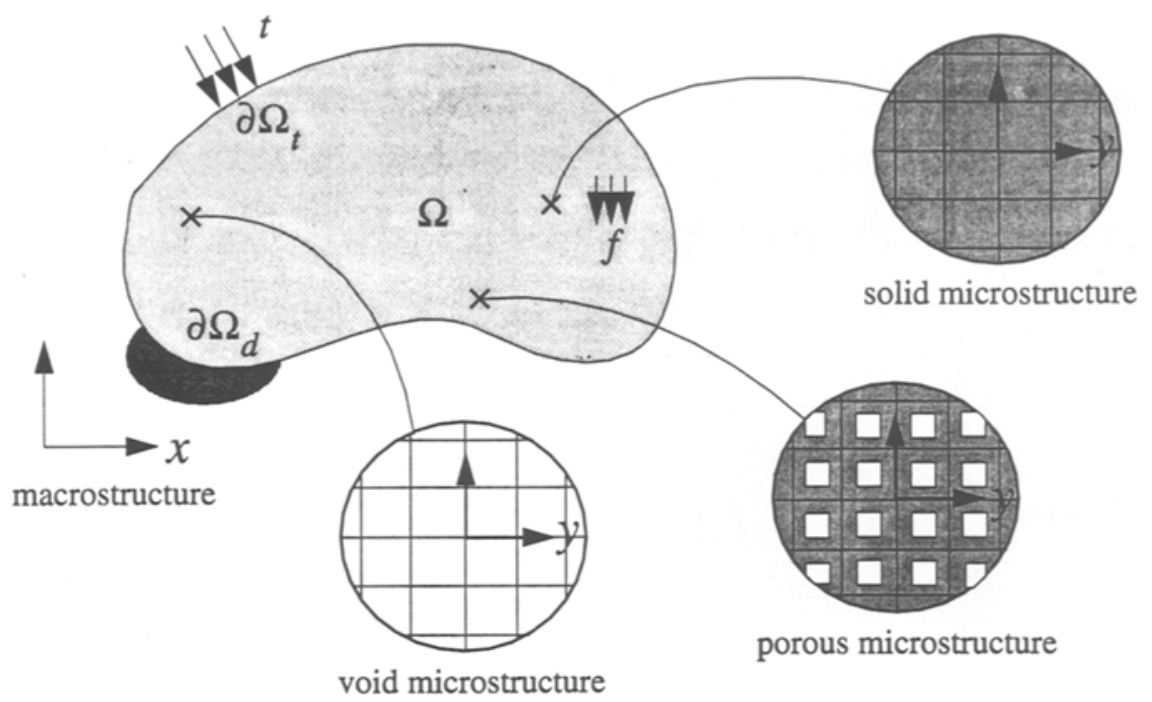

Fig. 1. Design domain and microstructures

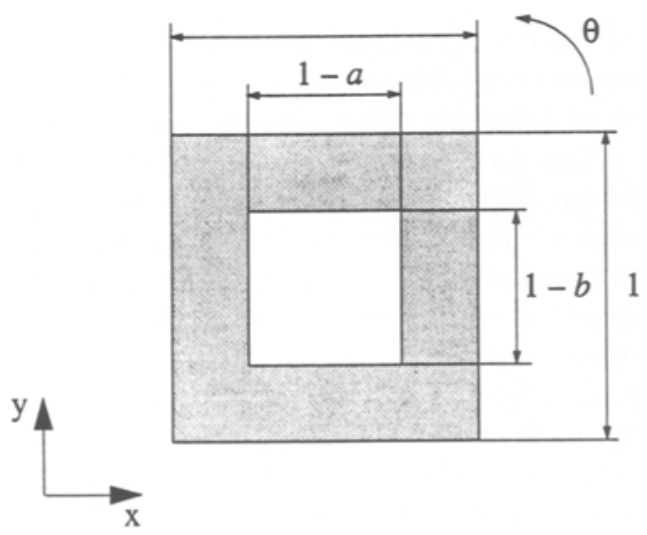

Fig. 2. Unit cell of a two-dimensional microstructure

able constraint, and the equilibrium equation. The optimality conditions are derived based on the optimality criteria method associated with the sensitivity analysis. The twodimensional as well as the three-dimensional plate and shell design problems are illustrated to substantiate this approach.

\section{Homogenization design method}

The homogenization design method (e.g. Bendsøe and Kikuchi 1988; Bendsøe 1989) entails finding the optimal material distribution within the elastic design domain while the criterion and constraints are satisfied. As shown in Fig. 1, the design domain $\Omega$ is composed of a porous material containing infinitely many microstructures, and the amount of material available is specified. In the design domain, boundary conditions are given and loading conditions, including the body force $f$ and the traction $t$ on the boundary $\partial \Omega_{t}$, are applied. The porosity of a microstructure is represented by a rectangular hole in a microstructure. A microstructure is classified as the void which contains no material (hole size $=$ 1), the solid medium which contains isotropic material (hole size $=0$ ), and the generalized porous medium which contains orthotropic material $(0<$ hole size $<1)$. The distribution of void, solid, and porous microstructures indicates the shape and topology of a structure.

A unit cell of a two-dimensional microstructure shown in Fig. 2 includes a rectangular hole of width $1-a$ and height $1-b$. The orientation of the material axes represents the rotation of the microstructure. The design variables are the size of the hole $D=\{a, b\}$ and the orientation of $\Theta=\{\theta\}$ the microstructure. The mass density of a microstructure is defined as $\rho=\rho_{0}(a+b-a b)$ where $\rho_{0}$ is the mass density of the isotropic material. Figure 3 illustrates a unit cell of threedimensional plate and shell microstructures. The unit cell consists of an isotropic base plate of thickness $h_{0}$ and two orthotropic reinforcement plates of thickness $h_{1}-h_{0}$ with rectangular holes. The size and rotation angle of the holes in the two reinforcement plates should be the same to avoid the coupling effect of bending and membrane deformation. The plate and shell model is developed using classical lamination theory which does not consider transverse shear deformations. Also, the structure of this element is approximated to be flat by means of projection onto the $x-y$ plane, in order to avoid the curvature effect. Thicknesses $h_{0}$ and $h_{1}$ are specified and the design variables are the same as in the two-dimensional problem under the plane stress assumption. If $\rho_{0}$ is the mass density of the isotropic material, the mass density of a microstructure is defined as $\rho=\rho_{0}\left[h_{0}+(a+b-a b)\left(h_{1}-h_{0}\right)\right]$.

Since the porosity is different over the design domain, the theory of homogenization is employed to evaluate equivalent elastic material properties of microstructures (Guedes and Kikuchi 1990). In homogenization theory, a structure is assumed to be composed of periodic microstructures, and the equivalent material properties are estimated by a limiting process that involves diminishing the microscopic size. In addition, the orientation of material axes must be considered to define material properties. Thus, elastic material properties of a structure can be defined by the dimensions and orientation of microstructural holes. During the optimization process, microstructures are changed between the void and the solid. This implies that material can be moved from 


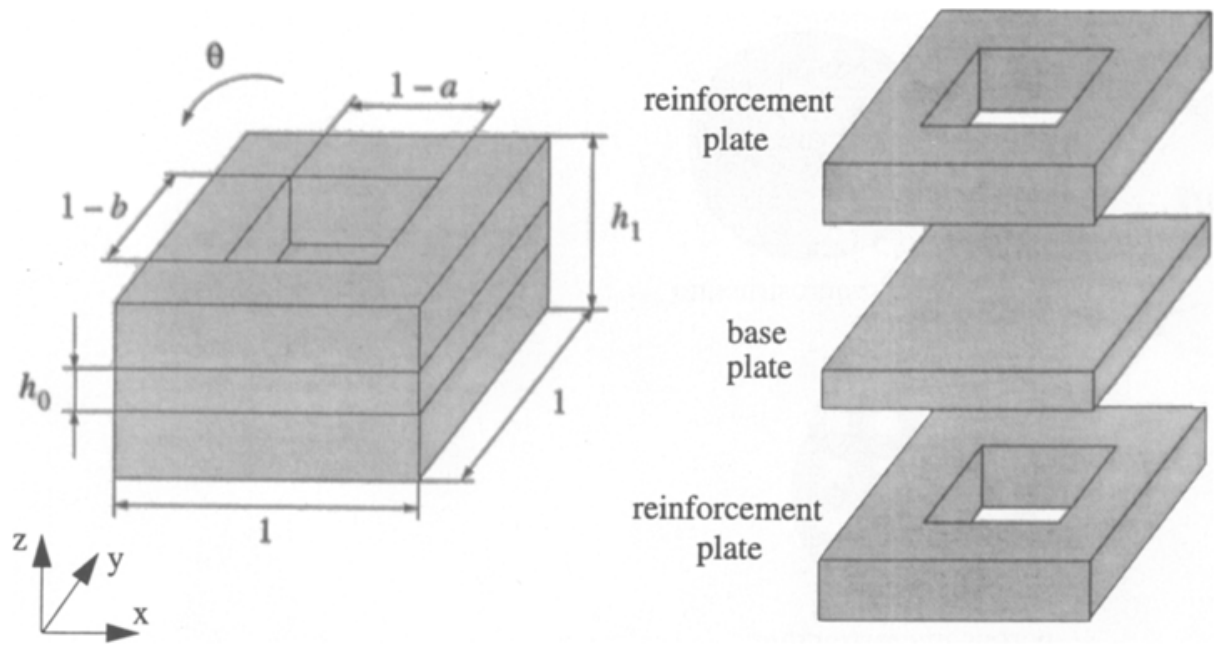

Fig. 3. Unit cell of a three-dimensional plate and shell microstructures

one part of the structure to another if the total amount of material available is specified. Thus, the optimal shape and topology design of structures can be regarded as finding an optimal material distribution within a prescribed admissible structural domain.

\section{Linear dynamic problem}

The equations of equilibrium governing the linear dynamic response of a system of finite elements can be written as

$\mathbf{M} \ddot{\mathbf{u}}+\mathbf{C} \dot{\mathbf{u}}+\mathbf{K u}=\mathbf{F}(t)$,

where $\mathbf{M}, \mathbf{C}$ and $\mathbf{K}$ are the mass, damping, and stiffness matrices, respectively; $\mathbf{F}(t)$ is the time-varying external load vector; and $\mathbf{u}, \dot{\mathbf{u}}$, and $\ddot{\mathbf{u}}$ are the displacement, velocity, and acceler ation vectors of the finite element assemblage, respectively. The procedures for the solution of the linear dynamic system can be divided into two methods: direct integration and mode superposition. In direct integration, the problem is solved by integrating the system of ordinary differential equations directly based on the appropriate finite difference approximation using a numerical step-by-step procedure. In mode superposition, the problem is solved by using eigenvalues and eigenvectors of the generalized free vibration problem defined by a reduced set of the basis functions. There is a concern about the reduced basis approximation since the response depends on the number of modes included in the mode superposition method. In this work, impact phenomena on structures are of interest and the linear transient response is obtained by using the direct integration method even though this method requires large computational effort.

The finite difference method for direct integration of a dynamic equilibrium equation can be categorized as explicit or implicit. In the explicit integration scheme, updated displacement vectors are determined in terms of completely historical information consisting of displacements and their time derivatives at the previous time step. It needs less core memory compared to the implicit scheme, but it is conditionally stable so that a small time step is required. This scheme is suitable for transient response problems of a structure rather than quasi-static and static problems. In the implicit integration scheme, computation of updated displacements requires information about the time derivatives of the updated displacement, which is unknown. It is unconditionally stable and has no restriction on the time step size other than as required for accuracy. But it is too expensive to solve large scale problems since we have to compute all matrices at each time step. It is considered that the explicit scheme is more practical in terms of the computational efforts than the implicit scheme in the case of the impact problem.

Here the central difference method is adopted as an explicit direct integration scheme because we are interested in the dynamic response within a very short period (Bathe 1996). It approximates velocity and acceleration by expanding $\mathbf{u}_{n+1}$ and $\mathbf{u}_{n-1}$ in Taylor series about time $t_{n}$ as

$\dot{\mathbf{u}}_{n}=\frac{1}{2 \Delta t}\left(\mathbf{u}_{n+1}-\mathbf{u}_{n-1}\right)$,

and

$\ddot{\mathbf{u}}_{n}=\frac{1}{\Delta t^{2}}\left(\mathbf{u}_{n+1}-2 \mathbf{u}_{n}+\mathbf{u}_{n-1}\right)$.

The displacement solution for time $t_{n+1}$ is obtained by considering (1) at time $t_{n}$

$\mathbf{M} \ddot{\mathbf{u}}_{n}+\mathbf{C} \dot{\mathbf{u}}_{n}+\mathbf{K} \mathbf{u}_{n}=\mathbf{F}_{n}(t)$.

Substituting the relations for $\dot{\mathbf{u}}_{n}$ and $\ddot{\mathbf{u}}_{n}$ in (2) and (3), respectively, into (4), we obtain

$$
\begin{aligned}
& \left(\frac{1}{\Delta t^{2}} \mathbf{M}+\frac{1}{2 \Delta t} \mathbf{C}\right) \mathbf{u}_{n+1}= \\
& \mathbf{F}_{n}-\left(\mathbf{K}-\frac{2}{\Delta t^{2}} \mathbf{M}\right) \mathbf{u}_{n}-\left(\frac{1}{\Delta t^{2}} \mathbf{M}-\frac{1}{2 \Delta t} \mathbf{C}\right) \mathbf{u}_{n-1},
\end{aligned}
$$

from which we can solve for $\mathbf{u}_{n+1}$. It should be noted that the solution $\mathbf{u}_{n+1}$ is based on using the equilibrium conditions at time $t_{n}$. If the Rayleigh damping, which is of the form

$\mathbf{C}=\alpha \mathbf{M}+\beta \mathbf{K}$, 


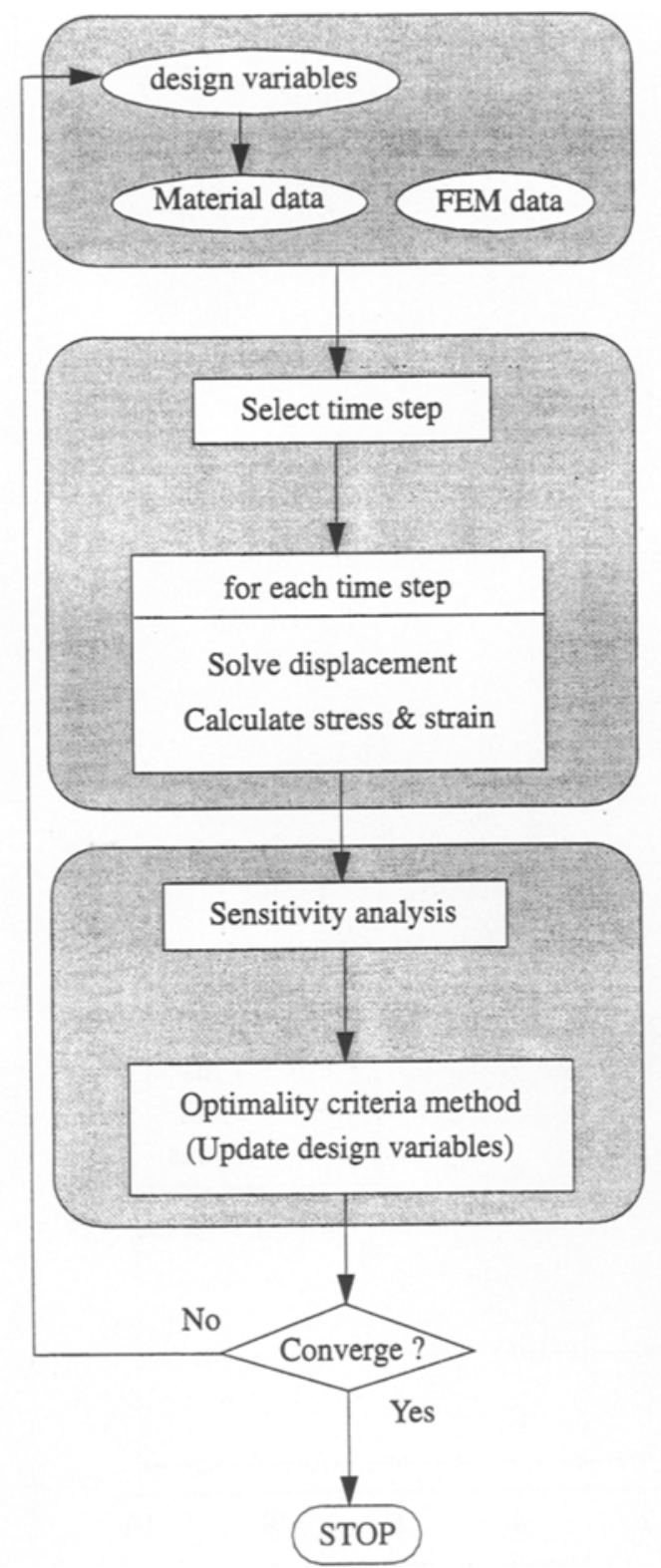

Fig. 4. Flow chart of the optimization procedure

is assumed, (5) can be written as

$\left[\left(\frac{1}{\Delta t^{2}}+\frac{\alpha}{2 \Delta t}\right) \mathbf{M}+\frac{\beta}{2 \Delta t} \mathbf{K}\right] \mathbf{u}_{n+1}=$

$\mathbf{F}_{n}-\left(\mathbf{K}-\frac{2}{\Delta t^{2}} \mathbf{M}\right) \mathbf{u}_{n-}$

$\left[\left(\frac{1}{\Delta t^{2}}-\frac{\alpha}{2 \Delta t}\right) \mathbf{M}-\frac{\beta}{2 \Delta t} \mathbf{K}\right] \mathbf{u}_{n-1}$

where $\alpha$ and $\beta$ are constants to be determined from two given damping ratios that correspond to two unequal frequencies of vibration.

Meanwhile, if a lumped mass matrix is assumed and the damping is neglected, then (5) can be reduced as

$\left(\frac{1}{\Delta t^{2}} \mathbf{M}\right) \mathbf{u}_{n+1}=$

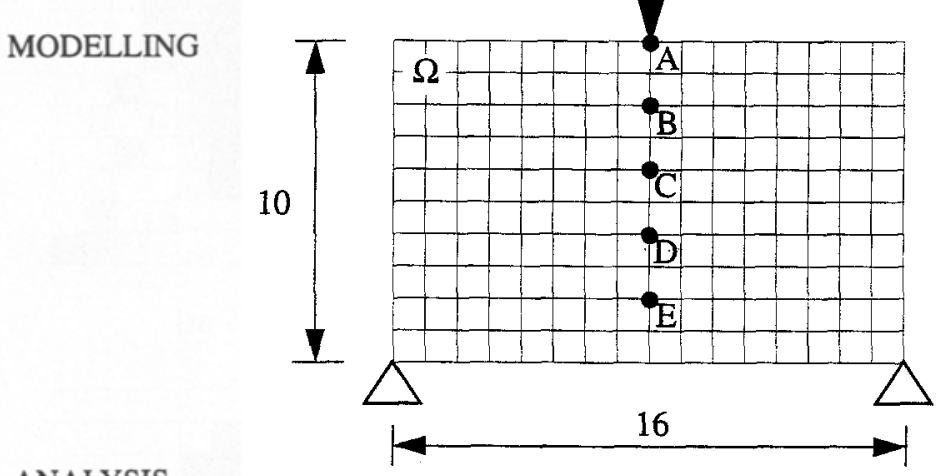

Fig. 5. Design domain of the 2D problem

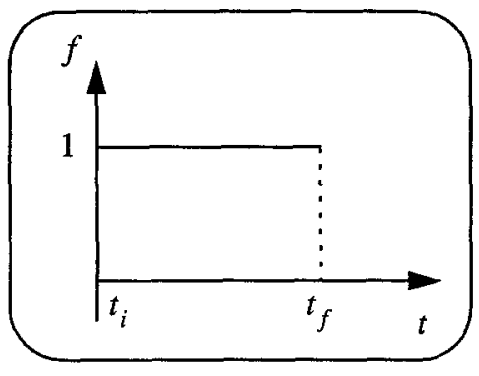

(a) case 1: step function

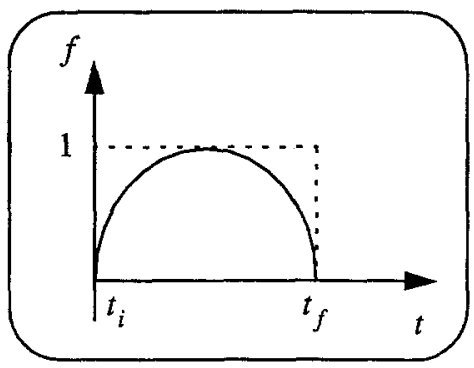

(b) case 2: sinusoidal function

Fig. 6. External force profiles. (a) case 1: step function; (b) case 2: sinusoidal function

$\mathbf{F}_{n}-\left(\mathbf{K}-\frac{2}{\Delta t^{2}} \mathbf{M}\right) \mathbf{u}_{n}-\left(\frac{1}{\Delta t^{2}} \mathbf{M}\right) \mathbf{u}_{n-1}$.

Therefore, the procedure to solve the displacement for time $t_{n+1}$ will be effective because only matrix multiplications are required without factorizing a matrix.

A consideration in the use of the central difference scheme is the stability because the integration method is conditionally stable, which means the time step should be smaller than a certain value, usually called the stability limit. Here the time step is determined in a practical sense by

$\Delta t \approx \frac{L_{\min }}{C_{d}}$ 


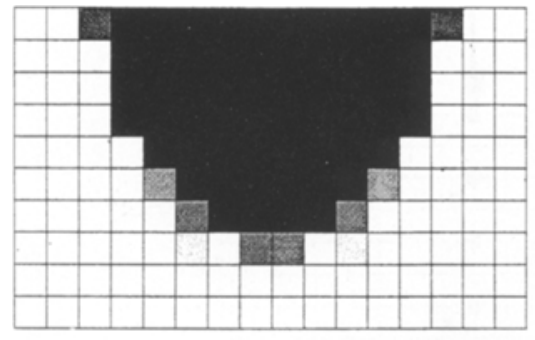

(a) $t_{f}=1 \mathrm{~ms}$

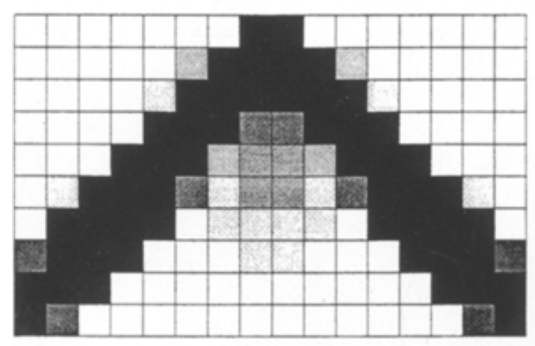

(c) $t_{f}=10 \mathrm{~ms}$

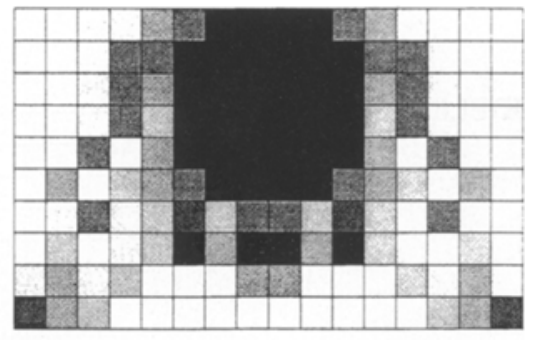

(b) $t_{f}=5 \mathrm{~ms}$

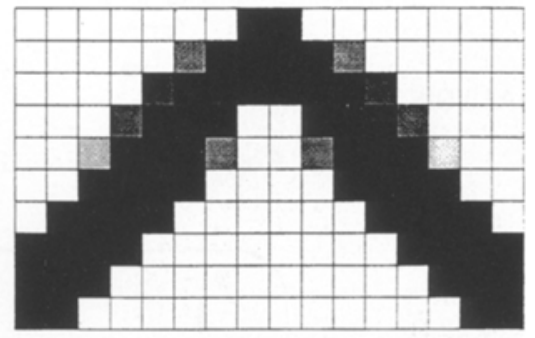

(d) static loading

Fig. 7. Optimal structural layouts of case 1

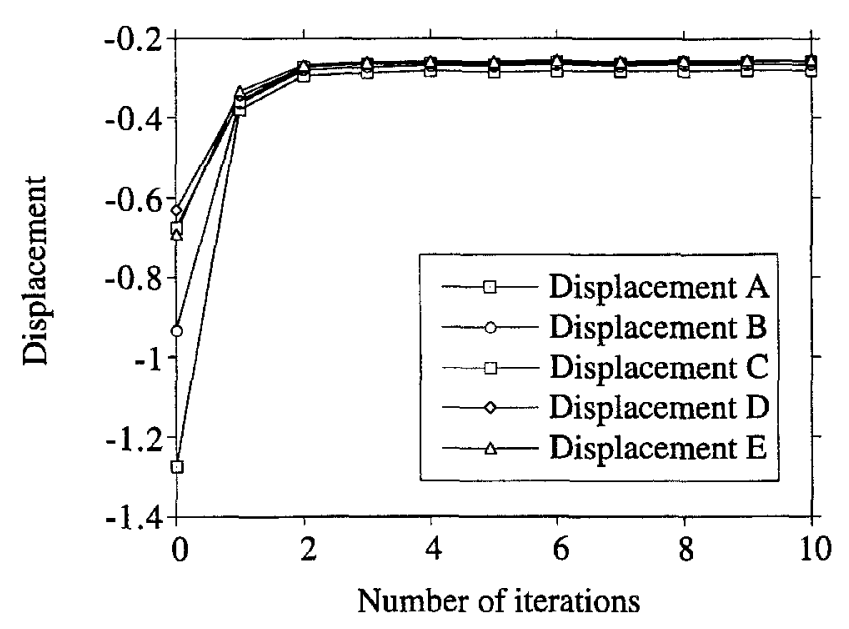

Fig. 8. Displacements in the optimization process $\left(t_{f}=5 \mathrm{~ms}\right)$

where $L_{\min }$ is the smallest lateral length in finite element mesh and $C_{d}$ is the dilatational wave speed of the material defined as

$C_{d}=\sqrt{\frac{\bar{\lambda}+2 \bar{\mu}}{\rho}}$

Here $\bar{\lambda}$ and $\bar{\mu}$ are the Lamé constants, and $\rho$ is the mass density.

A numerical step-by-step procedure using the central difference method can be summarized as follows.

- Form stiffness matrix $\mathbf{K}$, mass matrix $\mathbf{M}$, and damping matrix C.

- Initialize displacement $\mathbf{u}_{0}$, velocity $\dot{\mathbf{u}}_{0}$, and acceleration $\ddot{\mathbf{u}}_{0}$.

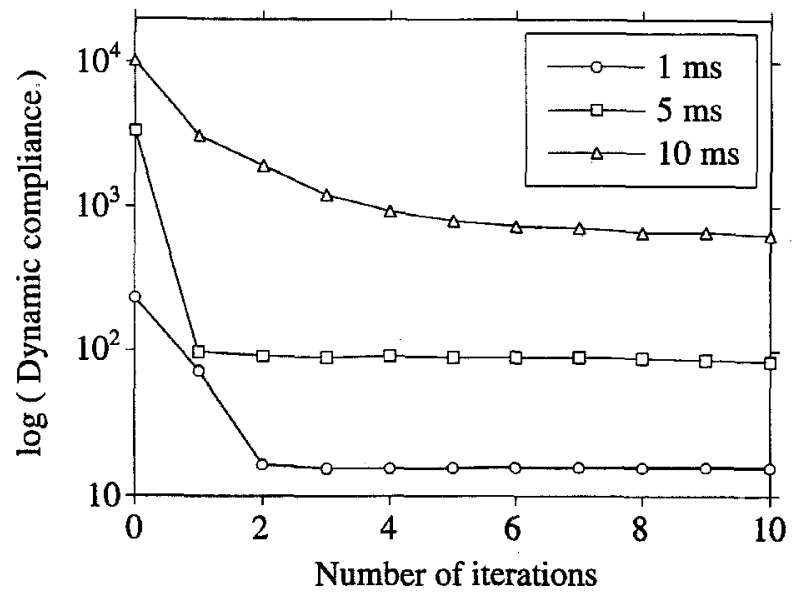

Fig. 9. Convergence history of the 2D problem

- Select the time step $\Delta t$.

- Calculate $\mathbf{u}_{-1}=\mathbf{u}_{0}-\Delta t \dot{\mathbf{u}}_{0}+\Delta t^{2} \ddot{\mathbf{u}}_{0} / 2$

- Calculate the right-hand-side of (5) at time $t_{n}$.

- Solve (5) for displacements at time $t_{n+1}$.

\section{Optimization problem}

The design goal of the optimization problem is to find the material distribution that minimizes the mean dynamic compliance of the structure under the dynamic loadings. The mean dynamic compliance of the structure during the time interval 


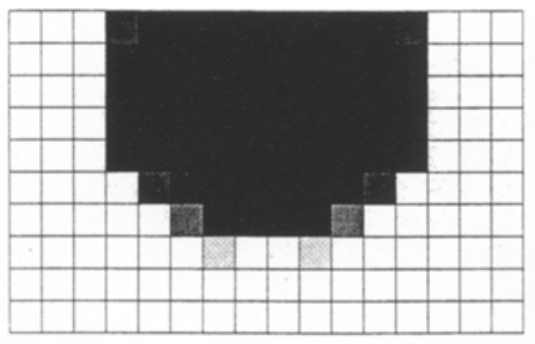

(a) $t_{f}=1 \mathrm{~ms}$

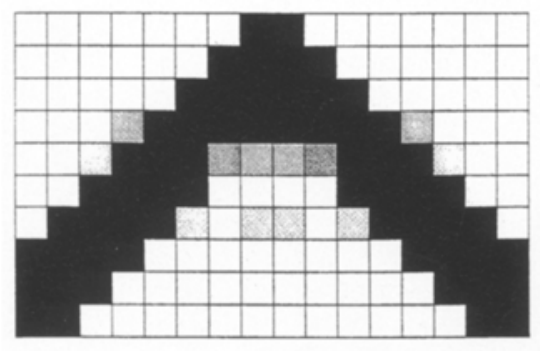

(c) $t_{f}=10 \mathrm{~ms}$

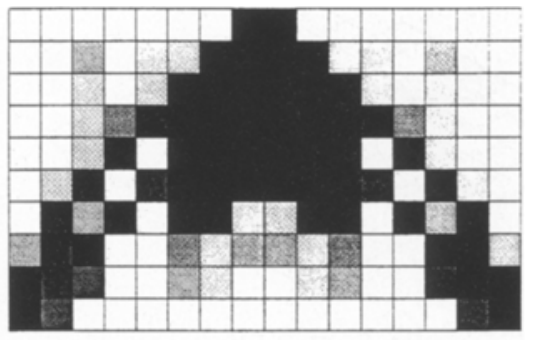

(b) $t_{f}=5 \mathrm{~ms}$

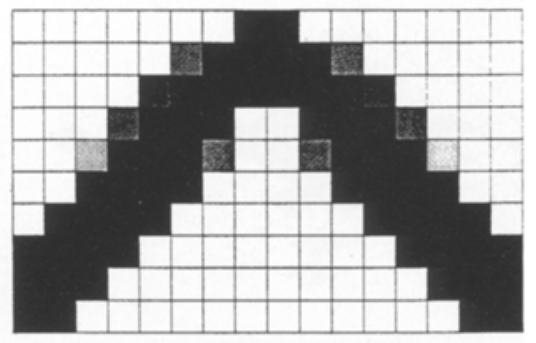

(d) static loading

Fig. 10. Optimal structural layouts of Case 2

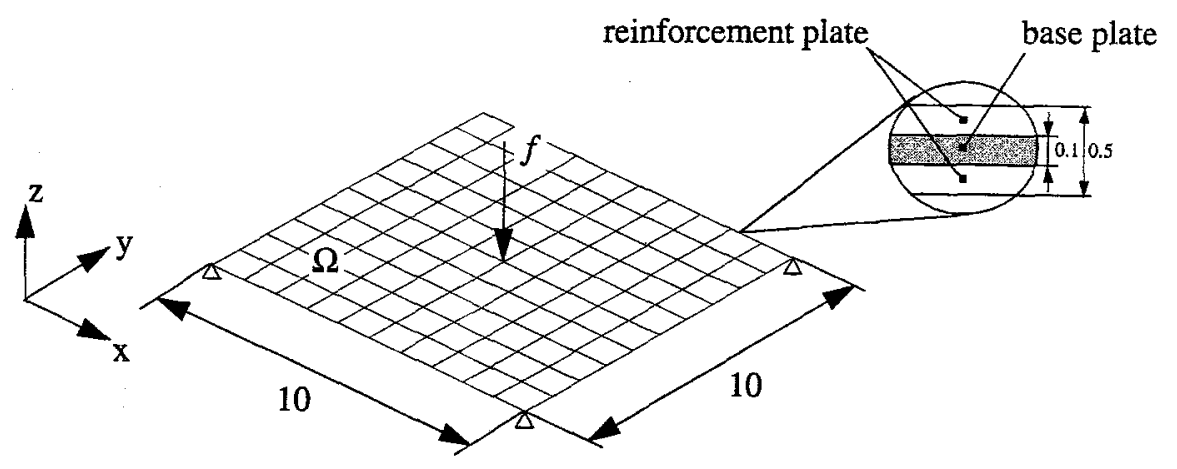

Fig. 11. Design domain of the plate problem

from the initial time $t_{i}$ to the final time $t_{f}$ can be defined as

$\Phi_{d}=\int_{t_{i}}^{t_{f}} \mathbf{u}^{T} \mathbf{F}(t) \mathrm{d} t$.

Thus, the optimization problem can be formulated using the finite element notion as follows.

Determine $D$ and $\Theta$ for a prescribed amount of material $\Omega_{s}$ that

$\min _{D, \Theta} f=\sum_{i=1}^{N_{t}} \sum_{i=1}^{N_{\text {dof }}} u_{i}^{T} F_{i} \Delta t$,

subject to the total mass constraint

$g=\sum_{i=1}^{N} \rho_{i} \Omega_{i}-\Omega_{s} \leq 0$, and the side constraint

$0 \leq d_{i}^{\ell} \leq d_{i} \leq d_{i}^{u} \leq 1$

including the equations of equilibrium. The design domain is discretized by $N$ finite elements which result in $N_{\text {dof }}$ degrees of freedom and the number of time steps $N_{t}$ is determined for the given time interval. Based on the finite element formulation, $F_{i}$ (the load vector of nodes in the $i$-th element) can be derived, and $u_{i}$ (the displacement of nodes in the $i$-th element) can be obtained by the linear dynamic analysis; $\rho_{i}$ and $\Omega_{i}$ stand for the mass density of a microstructure and the volume of the $i$-th element, while $d_{i}^{\ell}$ and $d_{i}^{u}$ represent the lower and the upper values of the size design variable, respectively.

Introducing a shift parameter $\mu$, necessary conditions for the optimality of design variables in the described optimization problem can be derived by defining the Lagrangian func- 


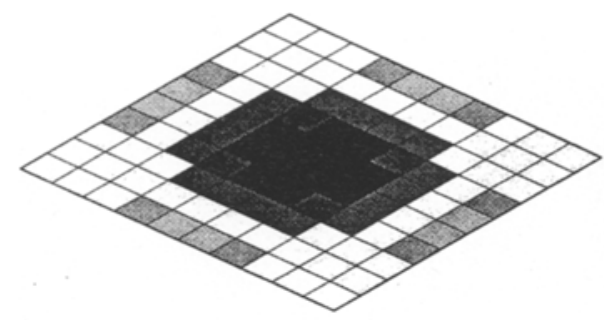

(a) $t_{f}=1 \mathrm{~ms}$

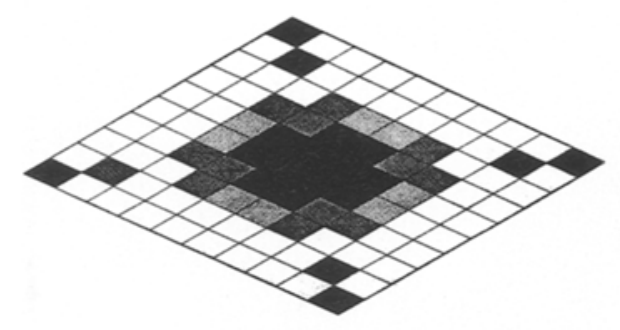

(c) $t_{f}=10 \mathrm{~ms}$

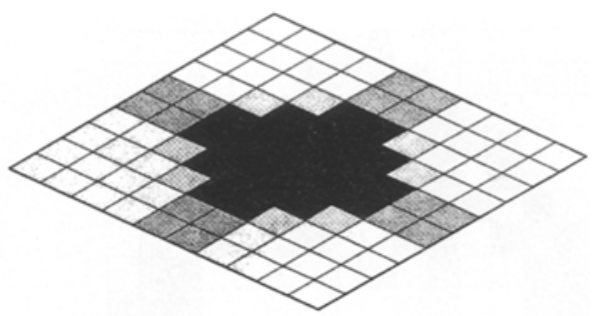

(b) $t_{f}=5 \mathrm{~ms}$

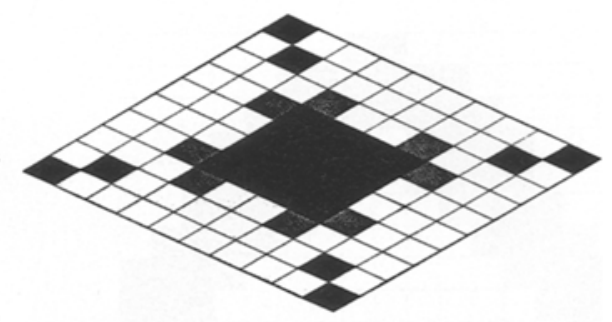

(d) static loading

Fig. 12. Optimal structural layouts of the plate problem
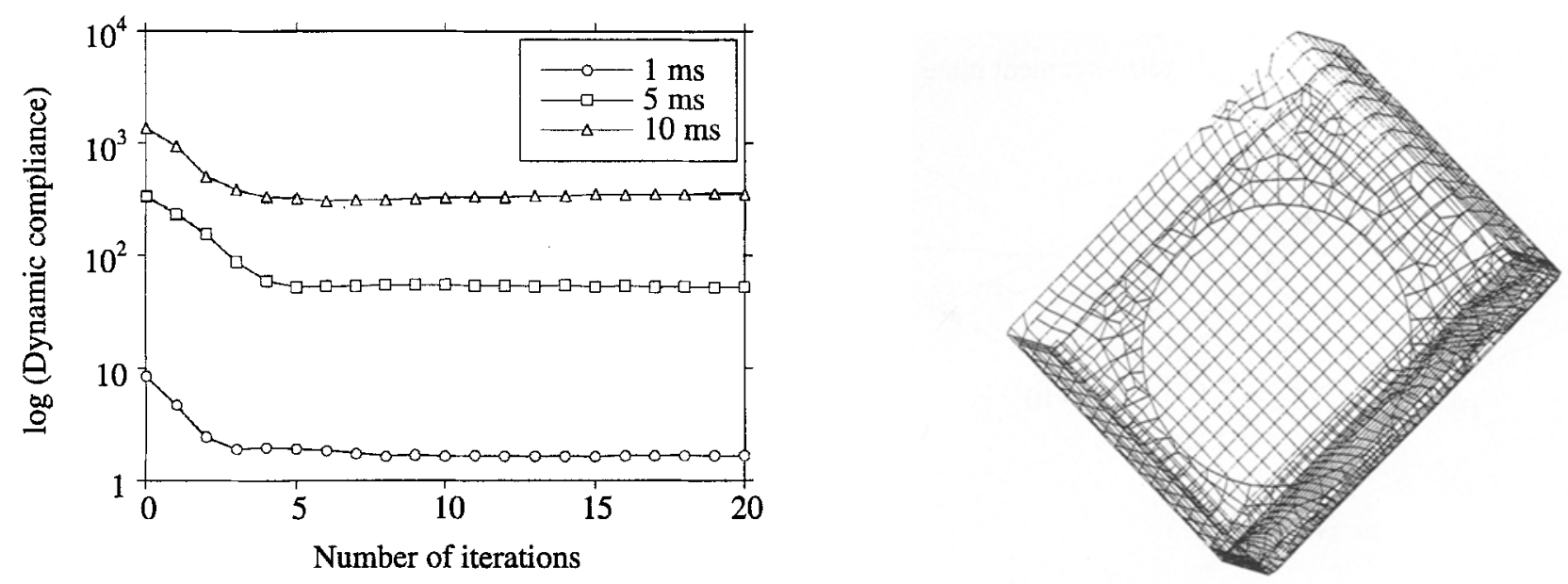

Fig. 13. Convergence history of the plate problem

tion

$L=(f-\mu g)+\tilde{\Gamma} g+\sum_{i=1}^{N_{d}}\left[\alpha_{i}^{\ell}\left(d_{i}^{\ell}-d_{i}\right)+\alpha_{i}^{u}\left(d_{i}-d_{i}^{u}\right)\right]$

where the shifted Lagrange multiplier $\tilde{\Gamma}$ implies $\tilde{\Gamma}=\mu+\Gamma ; \Gamma$, $\alpha_{i}^{\ell}$, and $\alpha_{i}^{u}$ are Lagrange multipliers of the constraints, and $N_{d}$ stands for the number of size design variables $\left(N_{d}=2 N\right.$ for $2 \mathrm{D}$, plate, and shell problems). Stationarity of $L$ with respect to design variables requires that

$\frac{\partial L}{\partial d_{i}}=\left(\frac{\partial f}{\partial d_{i}}-\mu \frac{\partial g}{\partial d_{i}}\right)+\tilde{\Gamma} \frac{\partial g}{\partial d_{i}}-\alpha_{i}^{\ell}+\alpha_{i}^{u}=0$,

for $i=1, \ldots, N_{d}$,

$\frac{\partial L}{\partial \theta_{i}}=\frac{\partial f}{\partial \theta_{i}}=0, \quad$ for $\quad i=1, \ldots, N_{\theta}$,

Fig. 14. Design domain of a box structure reinforcement

where $N_{\theta}$ stands for the number of orientation design variable ( $N_{\theta}=N$ for $2 \mathrm{D}$, plate, and shell problems), and complementary conditions. If the side constraint is inactive, that is, the size design variable belongs to the region between the lower and upper bounds, the multipliers $\alpha_{i}^{\ell}$ and $\alpha_{i}^{u}$ should be zero to satisfy the KKT conditions. Thus, (16) can be rewritten as

$e_{i}=\frac{1}{\tilde{\Gamma}}\left(\mu-\frac{\partial f}{\partial d_{i}} / \frac{\partial g}{\partial d_{i}}\right)=1, \quad$ for $\quad d_{i}^{\ell} \leq d_{i} \leq d_{i}^{u}$,

where $e_{i}$ is the effectiveness of the $i$-th design variable, and if the structure is not in the optimal stage, the effectiveness will be not equal to one. Here, the shift parameter is chosen to make the effectiveness positive, and multiplier $\tilde{\Gamma}$ is deter- 


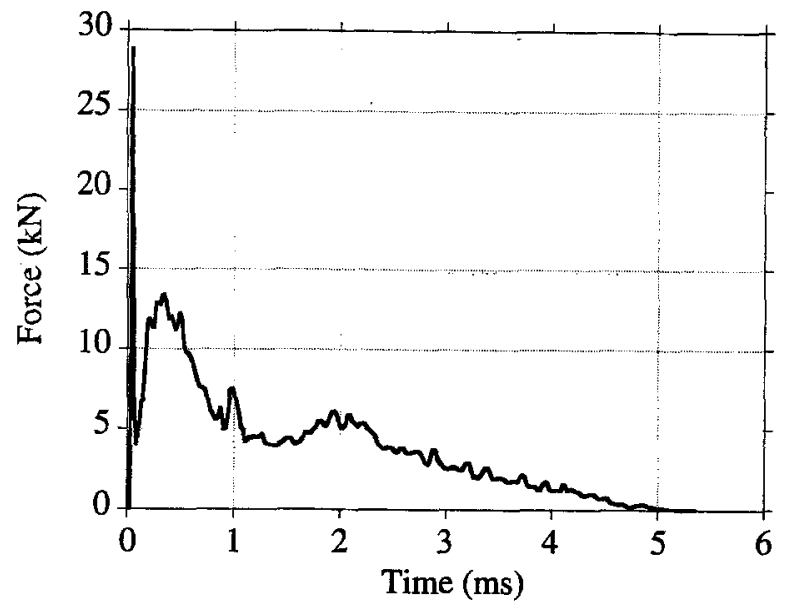

Fig. 15. Force-time data from PAMCRASH

mined by making the total mass constraint active. Therefore, the size design variables can be updated based on the modified optimality criteria method as follows:

$d_{i}^{k+1}=\left\{\begin{array}{lll}d_{i}^{\ell} & \text { if } \quad\left(e_{i}^{k}\right)^{\eta} d_{i}^{k} \leq d_{i}^{\ell} \\ \left(e_{i}^{k}\right)^{\eta} d_{i}^{k} & \text { if } \quad d_{i}^{\ell}<\left(e_{i}^{k}\right)^{\eta} d_{i}^{k} \leq d_{i}^{u} \\ d_{i}^{u} & \text { if } \quad d_{i}^{u} \leq\left(e_{i}^{k}\right)^{\eta} d_{i}^{k}\end{array}\right.$

where $d_{i}^{k}$ is the design variable in the $k$-th iteration, $d_{i}^{k+1}$ the updated design variable, and $\eta$ a weighting factor; $e_{i}^{k}$ is the effectiveness of the $i$-th design variable in the $k$-th iteration. Finally, the angle of rotation $\Theta$ should also be updated, using that the axes of orthotropy should align with the axes of principal stresses (Pedersen 1989).

The updating scheme for design variables requires the sensitivity information. The following sensitivity analysis is based on the hypothesis that the damping is neglected and a lumped mass matrix is considered. The sensitivity of the objective function at time $t_{n}$ can be written as

$\frac{\partial}{\partial d_{i}}\left(\mathbf{u}_{n}^{T} \mathbf{F}_{n}\right)=\frac{\partial}{\partial d_{i}}\left(\mathbf{u}_{n}^{T} \mathbf{M} \ddot{\mathbf{u}}_{n}+\mathbf{u}_{n}^{T} \mathbf{K} \mathbf{u}_{n}\right)=$

$\frac{\partial \mathbf{u}_{n}^{T}}{\partial d_{i}} \mathbf{M} \ddot{\mathbf{u}}_{n}+\mathbf{u}_{n}^{T} \frac{\partial \mathbf{M}}{\partial d_{i}} \ddot{\mathbf{u}}_{n}+\mathbf{u}_{n}^{T} \mathbf{M} \frac{\partial \ddot{\mathbf{u}}_{n}}{\partial d_{i}}+$

$\frac{\partial \mathbf{u}_{n}^{T}}{\partial d_{i}} \mathbf{K} \mathbf{u}_{n}+\mathbf{u}_{n}^{T} \frac{\partial \mathbf{K}}{\partial d_{i}} \mathbf{u}_{n}+\mathbf{u}_{n}^{T} \mathbf{K} \frac{\partial \mathbf{u}_{n}}{\partial d_{i}}$.

Once the acceleration sensitivity term is obtained by differentiating the equations of motion at time $t_{n}$ with respect to design variable as

$\mathbf{M} \frac{\partial \ddot{\mathbf{u}}_{n}}{\partial d_{i}}=-\frac{\partial \mathbf{M}}{\partial d_{i}} \ddot{\mathbf{u}}_{n}-\frac{\partial \mathbf{K}}{\partial d_{i}} \mathbf{u}_{n}-\mathbf{K} \frac{\partial \mathbf{u}_{n}}{\partial d_{i}}$,

the sensitivity of the objective function at time $t_{n}$ can be reduced as

$\frac{\partial}{\partial d_{i}}\left(\mathbf{u}_{n}^{T} \mathbf{F}_{n}\right)=\frac{\partial \mathbf{u}_{n}^{T}}{\partial d_{i}} \mathbf{M} \ddot{\mathbf{u}}_{n}+\frac{\partial \mathbf{u}_{n}^{T}}{\partial d_{i}} \mathbf{K} \mathbf{u}_{n}$ and the displacement sensitivity becomes the only unknown variable in the sensitivity analysis.

The displacement solution for time can be written as

$a_{0} \mathbf{M} \mathbf{u}_{n+1}=\mathbf{F}_{n}-\left(\mathbf{K}-a_{1} \mathbf{M}\right) \mathbf{u}_{n}-a_{1} \mathbf{M} \mathbf{u}_{n-1}$,

where $a_{0}=1 / \Delta t^{2}$ and $a_{1}=2 a_{0}$. Differentiating (23) with respect to design variable $d_{i}$ yields

$$
\begin{aligned}
& a_{0} \mathbf{M} \frac{\partial \mathbf{u}_{n+1}}{\partial d_{i}}=a_{0} \frac{\partial \mathbf{M}}{\partial d_{i}}\left(-\mathbf{u}_{n+1}+2 \mathbf{u}_{n}-\mathbf{u}_{n-1}\right)- \\
& \frac{\partial \mathbf{K}}{\partial d_{i}} \mathbf{u}_{n}-\left(\mathbf{K}-a_{1} \mathbf{M}\right) \frac{\partial \mathbf{u}_{n}}{\partial d_{i}}-a_{0} \mathbf{M} \frac{\partial \mathbf{u}_{n-1}}{\partial d_{i}} .
\end{aligned}
$$

Thus, the sensitivity of the objective function can be evaluated by plugging the solution of (24) into (22).

The overall procedure for the design optimization is illustrated in Fig. 4. In the optimization process, equations of motion and the sensitivity equations need to be integrated over time. This is an important step because most of the computational time is spent in numerical integration of these equations.

Table 1. Design improvement

\begin{tabular}{lll}
\hline & Initial design & Reinforcement design \\
\hline $\begin{array}{l}\text { Internal energy } \\
(\mathrm{kN} \mathrm{mm})\end{array}$ & 2 & 0.5 \\
$\begin{array}{l}\text { Kinetic energy } \\
(\mathrm{kN} \mathrm{mm})\end{array}$ & 0.7 & 0.3 \\
\hline
\end{tabular}

\section{$5 \quad$ Numerical examples}

The optimal topology design method which considers dynamic loadings is applied to the simple two-dimensional plane and three-dimensional plate problems to demonstrate the feasibility of this method. For the practical application, the reinforcement design problem is considered for a box-type structure under the drop test. In these examples, the material properties of the isotropic material are assumed as follows: Young's modulus $E=100 \mathrm{~N} / \mathrm{mm}^{2}$, Poisson's ratio $\nu=0.3$, and density $\rho_{0}=7.85 \times 10^{-6} \mathrm{~kg} / \mathrm{mm}^{3}$.

\subsection{Example 1: two-dimensional plane problem}

In this simple example, the rectangular area $\Omega$ shown in Fig. 5 is considered as the structural design domain in which the material will be distributed. The design domain is discretized by $16 \times 10$ QUAD4 finite elements and the impulsive-type dynamic loading is applied at the centreline of the top end. Two different kinds of dynamic load cases shown in Fig. 6 , the step function and the sinusoidal function, are applied to investigate the difference. Using the amount of material $\Omega_{s}=0.4 \Omega$, the optimization algorithm converges to the different solutions according to the different integration times ( $1 \mathrm{~ms}, 5 \mathrm{~ms}, 10 \mathrm{~ms}$, respectively). When the dynamic loading 


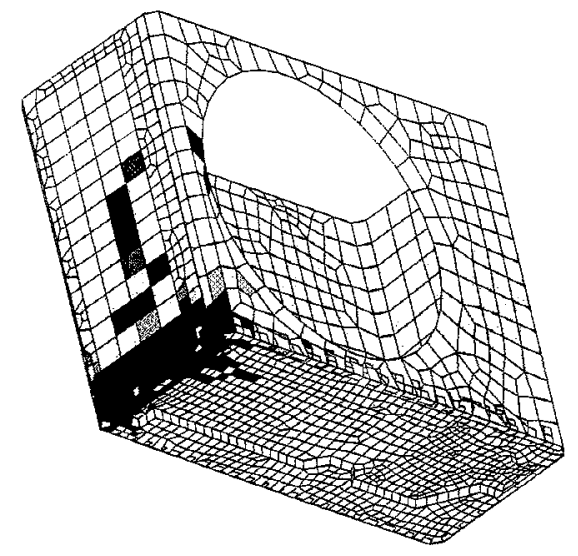

(a) $\Omega_{s}=0.25 \Omega$

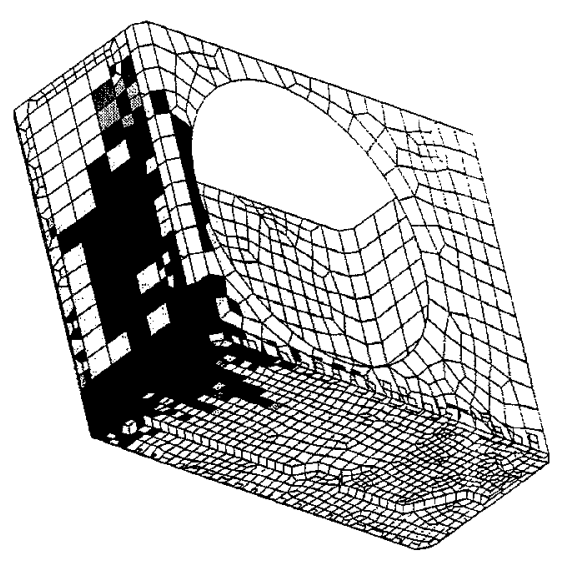

(b) $\Omega_{s}=0.30 \Omega$

Fig. 16. Optimal reinforcement design $\left(t_{f}=5 \mathrm{~ms}\right)$

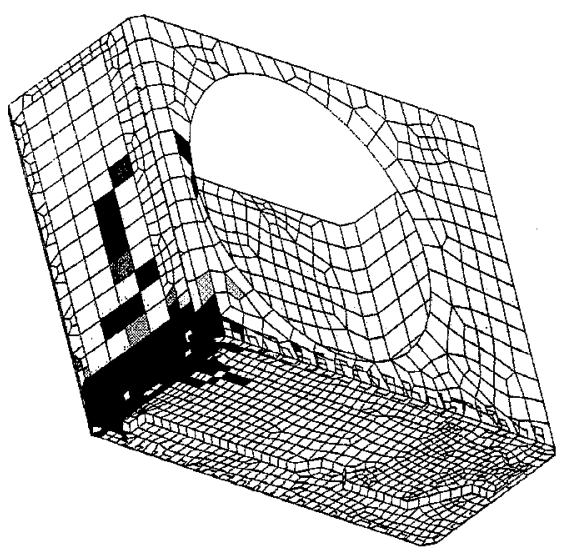

(a) $\Omega_{s}=0.25 \Omega$

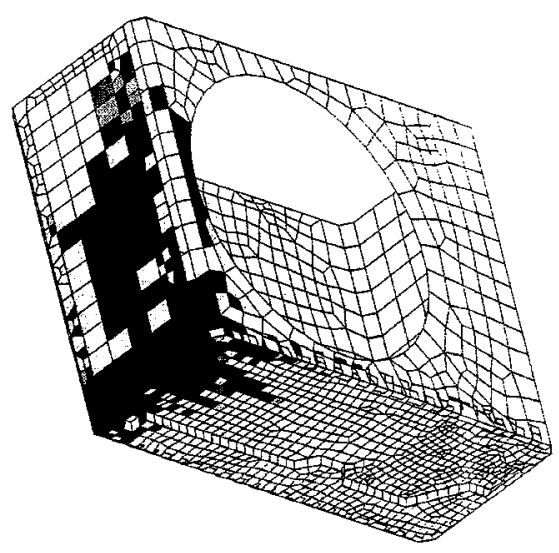

(b) $\Omega_{s}=0.30 \Omega$

Fig. 17. Optimal reinforcement design $\left(t_{f}=10 \mathrm{~ms}\right)$

is assumed as the step function (case 1), optimal layouts of the structure are shown in Figs. 7a-c. These layouts are compared to the one shown in Fig. $7 \mathrm{~d}$ generated from the optimal topology design problem for the static loading. It is noted that the optimal layout predicts the propagation of displacements and stresses in the structural domain. It is also shown that as the integration time increases, the optimal layout becomes similar to the one for the static loading, which implies the transition of the problem from the transient response to the steady state response. Figure 8 shows the history of the displacements at the location of A, B, C, D and E during the optimization process when the integration time is $5 \mathrm{~ms}$. It is seen that the displacements is reduced throughout the optimization, which implies the objective function tends to be minimized. Figure 9 illustrates the convergence history of the objective function, and it is observed that very fast convergence is achieved in this problem. In the case of the sinusoidal dynamic loading (case 2), similar optimal layouts are generated as shown in Figs. 10a-c. When compared to the static loading problem shown in Fig. 10d, it is shown that the optimal topology design under the time-varying dy- namic loads can produce the structural layout which predicts the propagation of the transient response.

\subsection{Example 2: three-dimensional plate problem}

A square plate simply supported at four corners shown in Fig. 11 is considered to be reinforced under the dynamic loading. The design domain $\Omega$ is discretized by $100(10 \times 10)$ QUAD4 finite elements and the impulsive load same as Fig. 6a is applied at the centre of the plate. The thickness of the base plate is assigned to 0.1 and the total thickness is set to 0.5 . Initially the design domain is assumed as uniform porosity of microstructures with $0.8 \times 0.8$ hole inside and no rotation. Using the amount of material $\Omega_{s}=0.4 \Omega$, the optimization algorithm distributes the material to minimize the dynamic compliance. Figures $12 \mathrm{a}-\mathrm{c}$ show that the material distribution for reinforcement converges to the different solutions depending upon the different integration times ( $1 \mathrm{~ms}, 5 \mathrm{~ms}, 10$ ms, respectively), and Fig. 12d shows that optimal topology design for the static loading. It is noted that the propagation of the deformation of a structure under time-varying loads 


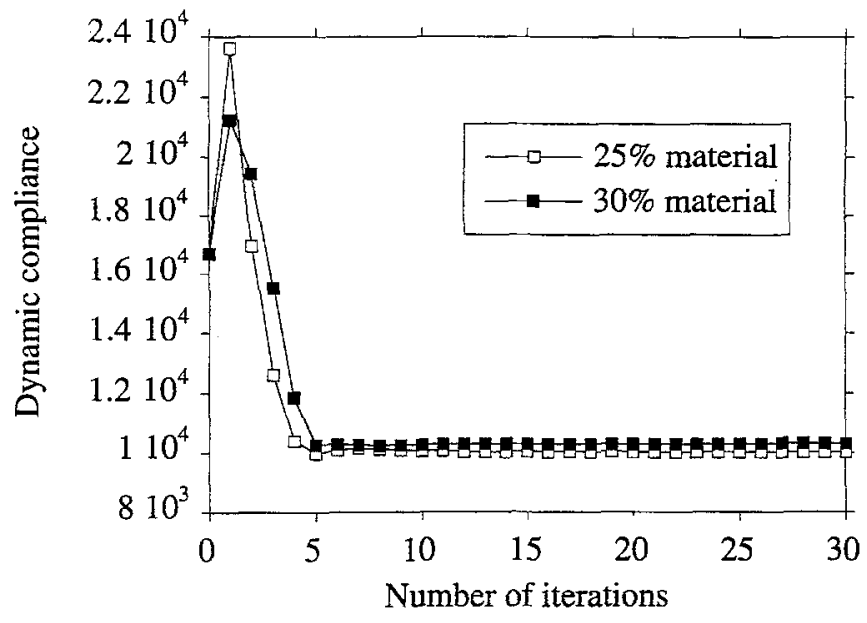

(a) objective function

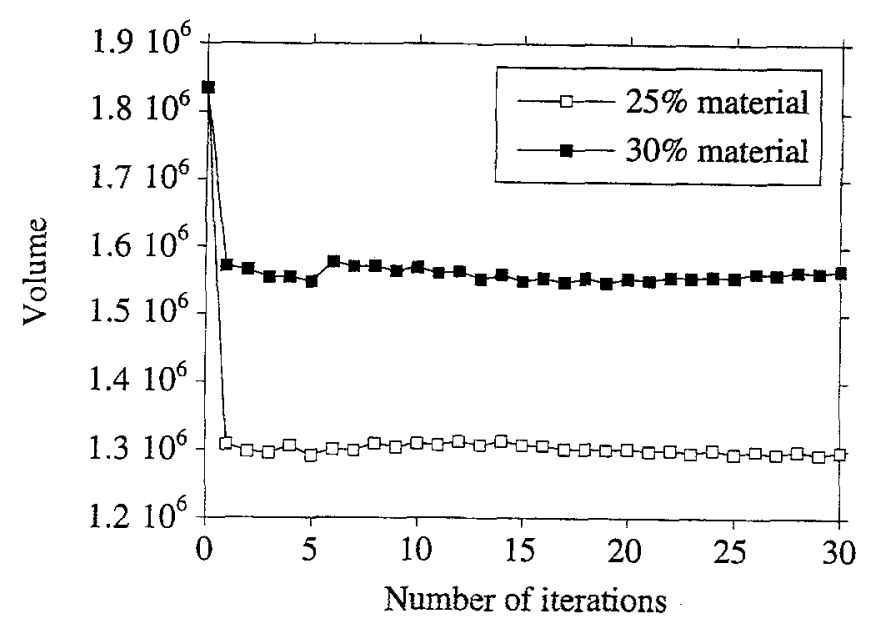

(b) constraint function

Fig. 18. Convergence history of example $3\left(t_{f}=5 \mathrm{~ms}\right)$

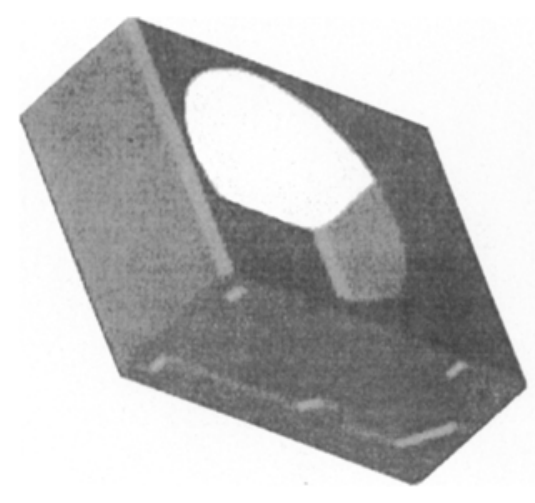

(a) initial design

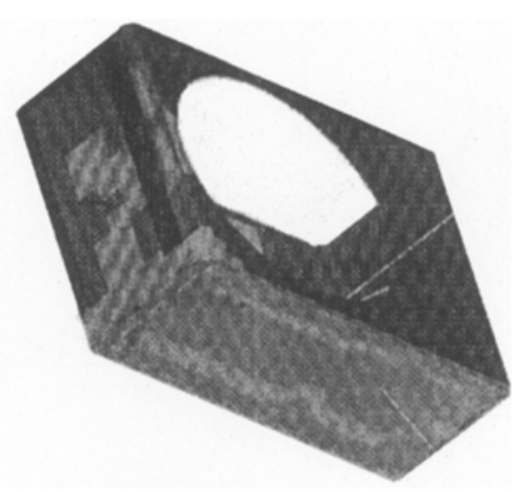

(b) reinforcement design

Fig. 19. Practical design based on the topology optimization

can be predicted based on the optimal topology design. The convergence history of the dynamic compliance is illustrated in Fig. 13 with different integration times

\subsection{Example 3: box structure reinforcement problem}

A box case structure shown in Fig. 14 is reinforced to minimize the dynamic compliance associated with the impact loads. The design domain is discretized into 2,326 fournode quadrilateral elements and 2,521 nodes. The time step is $5.08 \times 10^{-6}$ calculated based on the finite element size. The drop test is simulated by the commercial software PAMCRASH and the reaction force on the rigid wall with respect to time is shown in Fig. 15. These data are used to evaluate the response of the linear dynamic system. To deal with such a large-scale model efficiently, it is assumed that the inertia effect is so small enough to be neglected in the sensitivity analysis. Figure 16 shows the optimal reinforcement of the structure with different volume constraints considering the response during $5 \mathrm{~ms}$. It is seen that the material is distributed to the side of the base panel and the left panel, in which impact loads are applied, as the amount of material is increased. The optimal reinforcement design is proposed as shown in Fig. 17 when the integration time is extended to 10 ms with the same configuration. It is seen that the material tends to be concentrated to the part where impact loads are applied. The convergence history of both the objective function and the constraint in the case of $t_{f}=5$ is illustrated in Fig. 18. The optimal layout generated by the homogenization design method plays an important role to provide the design guideline at the concept initiation stage. Fig. 19 illustrates the initial design and the reinforcement design based on the optimal topology. Table 1 shows the performance improvement not only in the internal energy but also in the kinetic energy.

\section{Conclusions}

The homogenization design method extends its application to the optimal topology design of structures under time-varying dynamic loads. To analyze the transient structural response, an explicit direct integration scheme is implemented using the finite element model. The optimization problem is formulated to find the best configuration of structural systems that minimizes the dynamic compliance within the specified time interval, subject to the available material constraint. 
The updating scheme for design variables is derived by using an iterative algorithm based on the optimality criteria method. Results of numerical calculations demonstrate that the structural transient problem can be incorporated into the homogenization design method.

\section{References}

Bathe, K. J. 1996: Finite element procedures. Englewood Cliffs, NJ: Prentice-Hall

Bendsøe, M.P. 1989: Optimal shape design as a material distribution problem. Struct. Optim. 1, 193-202

Bendsøe, M.P.; Kikuchi, N. 1988: Generating optimal topologies in structural design using a homogenization method. Comput. Meth. Appl. Mech. Engrg. 71, 197-224

Cassis, J.H.; Schmit, L.A. 1976: Optimum structural design with dynamic constraints. J. Struct. Div. 102, 2053-2071
Chahande, A.I.; Arora, J.S. 1994: Optimization of large structures subjected to dynamic loads with the multiplier method. Int. J. Num. Meth. Engng. 37, 413-430

Fox, R.L.; Kapoor, M.P. 1970: Structural optimization in the dynamic regime: a computational approach. AIAA J. 8, 17981804

Guedes, J.M.; Kikuchi, N. 1990: Preprocessing and postprocessing for materials based on the homogenization method with adaptive finite element methods. Comput. Meth. Appl. Mech. Engrg. 83, 143-198

Pedersen, P. 1989: On optimal orientation of orthotropic materials. Struct. Optim. 1, 101-106

Pierson, B.L. 1972: A survey of optimal structural design under dynamic constraints. Int. J. Num. Meth. Engng 4, 491-499

Yamakawa, H. 1984: Optimum structural designs for dynamic response. In: Atrek, A.; Gallagher, R.G.; Ragsdell, K.M.; Zienkiewicz, O.C. (eds.) New directions in optimum structural design, pp. 249-266. New York: Wiley 